\title{
X-ray diagnosis of aseptic bone necrosis
}

\begin{abstract}
In the initial period of aseptic necrosis of bone radiography turns little informative, and patients get the treatment to neurologists with nevritami, pleksitami, miozitami and other nevrogennymi processes. Their therapy usually does not give effect. Only through 3-4 week occurs the characteristic bone structure change that clearly identifies repeated radiography and the patients are transferred to treatment to ortopedam-travmatologam, which hosts a variety of surgeries, of which best is closed excochleation foci of necrosis.
\end{abstract}

Keywords: radiography, bone, aseptic, necrosis, surgery
Volume 5 Issue 6 - 2018

\author{
Shaposhnikov Veniamin Ivanovich
}

Department of Oncology, Russia

Correspondence: Shaposhnikov VI, Department of Oncology with a course of radiation Diagnostics and therapy the NIGHT, Kuban Medical Institute, Krasnodar, Russia,

Email 79183445404@yandex.ru

Received: June 25, 20I8 | Published: November 26, 2018

\section{Introduction}

Despite the proliferation of no traumatic disorders of the musculoskeletal system, accompanied by aseptic necrosis and bone Lyses, this section of Orthopedics remains poorly studied. There is no clear idea about the etiopathogenesis, diagnosis and treatment of osteonekrozov. Spongy bone structures change often occurs when different arthritis and arthrosis of large joints, and are characterized by a certain clinical-radiological symptoms, while joint pathology is usually associated with a disease. Thus, in older women-menopause. ${ }^{1-4}$ Drivers of vehicles-with the vibration disease. ${ }^{3}$ But many causal factors, and for this reason are governed by polijetiologicheskim. It allows each factor to consider, as a degenerate-destructive process of bones that make up the large joints. ${ }^{1,5}$ Such consolidation allows you to develop, as a timely diagnosis and rational therapy of this disease.

This study is associated with the search method, which allows you to identify the aseptic necrosis of bone tissue at the early stages of the disease. Found that crucial in recognizing this illness has X-ray Diagnostics. In the development of pathological process, the authors distinguish 4 stages. 1-I-the contours of the head of the femur or humors bones, namely they are most often involved in the pathological process saved, but in chancellors bone can be seen ochazhki the destruction and sealing, 2-I-taped deformation contours of the head (usually the upper and the upper-outer) in the form of stairs. Articular crack extends through the development of reactive SYNOVITIS, article 3-plot clearly visible necrosis with symptoms of multiple sclerosis and bone growths in the articular cavity, 4-I shall head deformation with the formation of osteophytes in the cavity of joints. ${ }^{4,5}$ These bone structural changes are amplified. Joint function stops. The man turns to the still disabled. Thus, recognition of this disease in 1-th stage acquires great social importance, because current treatments allow you to correct this pathological process.

\section{The purpose of the work}

The study of diagnostic possibilities of radiography in recognition of aseptic bone necrosis, based on a correct interpretation of the clinical symptoms. And then perform closed hearth jekskohleaciju necrosis to remove harmful factors conducive to the development of bone destruction.

\section{Materials and methods}

Watched 856 patients with arthritis of large joints, distorting what is 13.6 to 10000 people. Out of the total number of these patients have been diagnosed with aseptic necrosis of 219(25.5\%). Of these, the head of the humerus was amazed at 144(65.7\%), head of femur-16(7.3\%), hip myshhelki-u30(13.7\%), ankle ankle-at 15(6.8\%), the myshhelki humerus-u $6(2.8 \%)$, the distal ends of the radius and ulna bones-8(3.7\%). The disease is most often seen in 41-60 years of age and had a one-sided localization; with the two sides was only $3 \%$ of patients. A history of trauma to the affected limb was noted in $63.3 \%$ of patients, and within reason, it evolved from $36.7 \%$. Duration of pathological process from 1 to 2 months was observed at $40(18.2 \%)$ the patients, from 3 to 6 months-62(28.3\%), up to 1 year-21(9.5\%), more than 1 year-96(43.5\%). All of these patients developed disease suddenly with the appearance of local pain in a joint without raising the local and overall body temperature. Immediately the patient's joint dysfunction occurred. Urgent was executed this joint radiography. The radiologist pathology is not found. Treatment of a neurologist about alleged neuritis. Limb function progressively worsened. In the shoulder and hip joints began to develop leading contracture of the upper or lower limbs. Required consultation of Orthopedic trauma. Condition of the patient was repeated radiography, which revealed hirakternuju pathology for aseptic necrosis. Retrospective analysis of the first radiographs showed the presence of primary signs of the pathological process in the form of mild osteoporosis cancellous bone and extending the joint cavity, which haven't been paying enough attention. The patient was made closed hearth excochleation necrosis on the author's methodology (RF Patent No. 2066138 dated September 10, 1996). The essence of the method is direct, vnesustavnaja trepanation bone awl or trochar. Once spike tool falls into a hotbed of necrosis from him under duress starts coming oily liquid. After the cessation, washed cavity $2 \%$ solution of hydrogen peroxide and furazilina solution. Then, curved bone spoon-sealed cavity by moving the sodrannyh fragments of border plots spongy and compact bone. After this hotbed of drained pout which is removed through 2-3, as by that time stops the selection of tissue detritus. After surgery, joint development begins immediately.

\section{Results \& discussion}

Postoperative complications were observed. Joint function quickly restored. It was found that the longer passed from the beginning of the disease and prior to surgery, the longer it took to restore limb function. In doing so, $63.9 \%$ of patients on treatment took up to 11 days, $24.2 \%$ from 12 to 20 days, at $8.2 \%$, from 21 to 30 days, $3.7 \%$ more than 1 month. Milestone radiography showed restoring bone structure (Figure 1a-1c). 


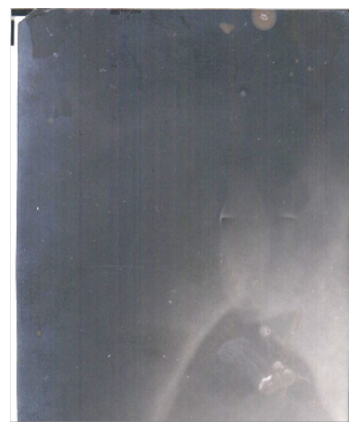

(a)

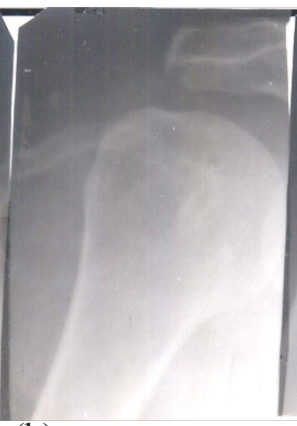

(b)

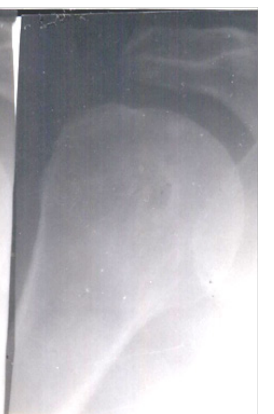

(c)

Figure Ia Radiograph Aseptic necrosis of large humerus. b: Radiograph operation completed site of with its sealing and drainage. c: Radiograph Completed treatment structure of the bone restored.

\section{Conclusion}

Aseptic necrosis of bone tissue can be traced to a common pathology in persons of mature age. He is accompanied by a characteristic clinical picture. The leading value in recognizing this illness has radiography. Early diagnosis allows you to quickly

abolish the pathological process through the use of a closed hearth jekskohleacii necrosis.

\section{Acknowledgments}

None.

\section{Conflicts of interest}

The author declares that there is no conflicts of interest.

\section{References}

1. Astapenko MG. Joint diseases. Medicine. 1966:379.

2. Benevolenskaya LI. Osteoporosis. Diagnosis, prevention and treatment. Clinical guidelines. 2005:171-181.

3. Krupko IL. Guide to traumatology and orthopedics. Medicine. 1975:271.

4. Rozhinskaya L Ya. Postmenopausal and senile osteoporosis: modern possibilities of diagnosis, prevention and treatment. Consilium-medicum. 2003;12(5):67-70.

5. Sokov LP. Deforming arthrosis of large joints. Peoples' Friendship University Press. 1991:119. 